\title{
Luminosity Correlations, Luminosity Evolutions, and Radio Loudness of AGNs from Multiwavelength Observations
}

\author{
Vahe Petrosian, Jack Singal and Lukasz Stawarz \\ Stanford University, CA, USA \\ Email: vahep@stanford.edu
}

\begin{abstract}
The dichotomy of jet dominated versus accretion disk dominated AGNs or "radioloud" vs "radio-quiet" quasars can be investigated by a simultaneous determination of the relative shape and evolution of the radio and optical luminosity functions, and the distribution of the radio loudness $\mathrm{R}$ defined as the ratio of radio to optical luminosities. This can be done from a multivariate data set containing observed fluxes, redshift, spectra, etc. We emphasize that when dealing with a multivariate data set it is imperative to first determine the true correlations, not those introduced by the observational selection effects, among the variables (e.g. Luminosityluminosity, redshift-luminosity) before obtaining the individual distributions of the variables (e.g. Luminosity functions and density evolution). We use data from several sources including the SDSS (Data Release 7) and FIRST radio catalogs, with well defined optical and radio flux limits, and employ the non-parametric methods developed by Efron and Petrosian, designed to obtain unbiased correlations, distributions and evolution with redshift from data truncated due to observational biases. We determine the density and the luminosity evolutions in both wavebands, which shows significantly higher radio than optical luminosity evolution. From these we obtain true distribution of the radio loudness parameter which shows no sign of bi-modality and indicates that quasars were more radio loud at earlier epochs.
\end{abstract}

